\title{
ESTUDIO DE LA CONSTRUCCIÓN DE ÍNDICES INTEGRALES PARA EL APOYO AL CONTROL DE GESTIÓN EMPRESARIAL
}

\author{
Alberto Medina León ${ }^{1}$, Neydalis Piloto Fleitas, Dianelys Nogueira Rivera, Arialys Hernández Nariño, Alejandro \\ Ricardo Alonso y Jorge Viteri Moya ${ }^{2}$.
}

\begin{abstract}
RESUMEN
El trabajo intenta una recopilación de los índices integrales realizados en Cuba como apoyo a la gestión empresarial en los últimos años. Sobre la base del estudio de estos indicadores se concluye, sustentada en la similitud de los enfoques empleados, con la elaboración de un procedimiento que sintetiza las experiencias de cerca de 30 indicadores; así como resume los métodos y herramientas empleadas por los diversos autores en las distintas etapas, donde se aprecia una notable diversidad de enfoques en el abordaje de las investigaciones. Además, se brindan consideraciones acerca de la importancia de esta tendencia de gestión en nuestras condiciones actuales, a la vez que valoraciones de posibles mejoras a incorporar en la obtención de esta herramienta.
\end{abstract}

\begin{abstract}
This work intends to do a comprehensible compilation of integrated indexes created in Cuba the last years, as a supporting tool to organizations management systems. Based on the study of 30 indexes, authors reach to conclusions, sustained in similar attributes of the compiled approaches, the consequent design of a methodology that synthesizes the experiences of such tools together with the illustration of methods and techniques employed in the different phases of the proposed methodologies. This analysis shows a considerable diversity of approaches used in the researches. Besides, the article offers considerations about the importance of this management practice in our particular environment as well as suggestions to improve the design of those instruments.
\end{abstract}

\footnotetext{
${ }^{1}$ Universidad de Matanzas Camilo Cienfuegos, Cuba

2 Universidad Tecnológica Equinoccial, Facultad de Ciencias de la Ingeniería, Av. Occidental y Mariana de Jesús. Quito, Ecuador.jviteri@ute.edu.ec
} 


\section{INTRODUCCIÓN}

El Control de Gestión debe responder a las necesidades de autonomía, mentalidad de eficiencia, ahorro y creatividad de las empresas. Adecuadamente empleado, permite vigilar el progreso, rectificar los errores, seguir los cambios del entorno y las repercusiones que estos producen en el avance de la organización, hacer los ciclos más rápidos, agregar valor a los productos y servicios de la empresa, unir a los trabajadores (independientemente de sus antecedentes y culturas), delegar y trabajar en equipo; Nogueira Rivera, D, \& Medina León, A. (2003).

El Control de Gestión se posiciona en un plano cuyas aristas se mueven en tres dimensiones: la estratégica, la operativa y la económica. A través de él, la empresa configura sus decisiones estratégicas, mediante el análisis del entorno y las posibilidades y potenciales propias de la organización, evalúa la implementación de las decisiones de forma operativa, verifica el cumplimiento de los procedimientos y procesos y realiza los análisis económicos, sobre la base de un sistema informativo (SI), eficiente, oportuno y eficaz, que permita la corrección de las desviaciones y su seguimiento (Nogueira Rivera et al., 2004).

\section{METODOLOGÍA}

- Análisis y de síntesis: Para detallar los procesos de diseño, integración, implementación, operación de los criterios y herramientas utilizadas, manifiesto en la división mental del todo en sus múltiples relaciones componentes y lograr establecer mentalmente la unión entre las partes, previamente analizadas para descubrir relaciones y características generales de los elementos de la realidad.

- Inducción y de deducción: Se aprecia al definir como una forma de razonamiento por medio de la cual se pasa del conocimiento de cosas particulares a un conocimiento más general que refleja lo que hay de común en los fenómenos individuales.

- Análisis histórico y el lógico: Se manifiesta en la trayectoria real de los fenómenos y acontecimientos en la evolución de su historia: Índices Integrales y su forma de obtención.

- Enfoque en sistema: Contemplado en la orientación general para el estudio de los fenómenos como una realidad integral formada por componentes, que cumplen funciones y mantienen formas estables de interacción entre ellos.

- Métodos Estadísticos: Resultan las herramientas de apoyo a la propuesta metodológica planteada. 


\section{RESULTADOS Y DISCUSIÓN}

El éxito del Control está en su utilización para la toma de decisiones y transita por la correcta definición de las variables que condicionan los resultados, la determinación de indicadores, su medición y posterior comparación con los parámetros fijados o deseados. La determinación correcta de indicadores resulta una actividad compleja, a la vez que actual e importante como se manifiesta en conjugación con la filosofía del Cuadro del Mando Integral.

El uso de indicadores sintéticos, para evaluar la eficiencia del sistema, ha cobrado una amplia difusión en los últimos tiempos en Cuba e Internacionalmente, a juicio de Medina León et al. (2005) por las razones siguientes:

- el resultado es un único valor que permite una fácil comparación con períodos precedentes u otras empresas; así como el estudio de tendencias,

- los elementos contemplados en su creación son producto de un estudio científicamente argumentado, en el cual resultaron fuentes de la información académicos y empresarios reconocidos en la actividad que se estudia,

- no requiere que los empresarios tengan de un conocimiento profundo sobre el tema que aborda el indicador para su utilización en la toma de decisiones,

- permiten ser automatizados fácilmente,

- resulta factible crear una relación causa - efecto entre los resultados alcanzados y los inductores de actuación; $y$

- son herramientas que permiten un diagnóstico permanente del sistema, así como vincularse a otros procedimientos de mejora empresarial.

Medina León et al. (2005) plantea que entre las limitaciones más comunes a estos indicadores integrales, se pueden citar:

- escasa utilización de software asociados a los indicadores integrales, a pesar de las facilidades que estos brindan para su automatización, y aún menor, el vínculo de los software creados a los sistemas informatizados existentes en las empresas,

- no se fijan criterios de evaluación para los elementos que forman parte de los indicadores, lo que implica evaluaciones no homogéneas en su aplicación,

- evalúan el sistema, limitados a diagnosticar los principales elementos provocadores de las desviaciones y no a incidir sobre sus inductores de actuación; y

- limitado uso del carácter proactivo. 
Entre los Indicadores Integrales creados en Cuba en los primeros años del presente siglo se encuentran: Suárez Mella, 1995 y 2001 para la Excelencia Organizativa Industrial (EOI); Hernández Maden, 1999 respecto al Nivel de Excelencia de la Distribución (EOD); Brito Viñas, 2000 con los Índices de Capacidad y Gestión de la Capacidad Tecnológica; Nogueira Rivera, 2002, Nogueira Rivera et al. (2002), para el Índice de Eficiencia Financiera (IEF); Negrín Sosa, 2003 en la Excelencia Organizativa Hotelera $(E O H)$ y la Excelencia Organizativa de los Procesos $(E O P)$, entre otro sin pretender ser conclusivo.

Esta tendencia ha continuado su proliferación en investigaciones más recientes. Un intento de relacionar los Índices Integrales elaborados en Cuba para el apoyo a la gestión Empresarial se muestra en la tabla 1.

Un estudio a profundidad de la construcción de los Índices Integrales muestra la utilización de un conjunto de pasos comunes, los que se pueden resumir en:

1. Recopilación de los síntomas.

2. Reducción del listado.

3. Obtención de coeficientes de prioridades (pesos) por métodos multicriterio.

4. Formulación matemática y cálculo del índice cuantitativo.

En contradicción a la homogeneidad de los pasos para la elaboración de los índices, se aprecia una amplia diversidad de métodos empleados en cada uno para dar solución a los objetivos deseados.

Tabla 1. Índices integrales creados en Cuba.

\begin{tabular}{|c|c|c|c|}
\hline Nombre & Autor & Publicación & Año \\
\hline $\begin{array}{l}\text { Índice de Excelencia Organizativa In- } \\
\text { dustrial }(E O I)\end{array}$ & Rogelio Suárez Mella & Tesis Doctoral & 1995 \\
\hline $\begin{array}{l}\text { Índice de Gestión de la Capacidad Tec- } \\
\text { nológica (CGI) }\end{array}$ & $\begin{array}{l}\text { Beatriz Cristina Brito } \\
\text { Viñas }\end{array}$ & Tesis Doctoral & 2000 \\
\hline Índice de Inconsistencia Global (IIG) & $\begin{array}{l}\text { Fernando Marrero Del- } \\
\text { gado. }\end{array}$ & Tesis doctoral. & 2001 \\
\hline $\begin{array}{l}\text { Nivel de Efectividad del Funcionamien- } \\
\text { to de la Cadena Logística }(\text { NEFCL) }\end{array}$ & $\begin{array}{l}\text { Fernando Marrero Del- } \\
\text { gado. }\end{array}$ & Tesis doctoral. & 2001 \\
\hline $\begin{array}{l}\text { Índice Integral de Eficiencia Financiera } \\
(I I E F)\end{array}$ & $\begin{array}{l}\text { Dianelys Nogueira Rive- } \\
\text { ra }\end{array}$ & Tesis doctoral. & 2002 \\
\hline Índice de Experticidad (IE) & Oñate Ramos & UCLV & 2002 \\
\hline
\end{tabular}




\begin{tabular}{|c|c|c|c|}
\hline Índice de Efectividad & $\begin{array}{l}\text { Clara Elena Marrero } \\
\text { Fornaris }\end{array}$ & Tesis Doctoral & 2002 \\
\hline Índice de Eficiencia & $\begin{array}{l}\text { Clara Elena Marrero } \\
\text { Fornaris }\end{array}$ & Tesis Doctoral & 2002 \\
\hline $\begin{array}{l}\text { Excelencia de los Procesos Hoteleros } \\
(\boldsymbol{E P H})\end{array}$ & Ernesto Negrín Sosa & Tesis doctoral. & 2003 \\
\hline $\begin{array}{l}\text { Nivel de Acercamiento a los Deseos de } \\
\text { los Clientes que Alcanza una Empresa } \\
m(N A D C m)\end{array}$ & $\begin{array}{l}\text { William Ariel Sarache } \\
\text { Castro }\end{array}$ & Tesis doctoral. & 2003 \\
\hline $\begin{array}{l}\text { Excelencia de los Procesos Hoteleros } \\
(\boldsymbol{E P H})\end{array}$ & Ernesto Negrín Sosa & Tesis doctoral. & 2003 \\
\hline $\begin{array}{l}\text { Intensidad Innovadora en la Empresa } \\
\text { Ganadera (IIEG) }\end{array}$ & Jesús Suárez Hernández & Tesis Doctoral & 2003 \\
\hline $\begin{array}{l}\text { Nivel de Excelencia en Empresas Gana- } \\
\text { deras }(\boldsymbol{E} O \boldsymbol{E} G)\end{array}$ & Jesús Suárez Hernández & Tesis Doctoral & 2003 \\
\hline $\begin{array}{l}\text { Nivel de Excelencia de la Tecnología en } \\
\text { la Empresa (ETE) }\end{array}$ & Jesús Suárez Hernández & Tesis Doctoral & 2003 \\
\hline $\begin{array}{l}\text { Índice del Nivel de Desempeño y/o } \\
\text { Actuación Competitiva (INAd) }\end{array}$ & Santiago Ibarra Mirón & Tesis Doctoral & 2003 \\
\hline $\begin{array}{l}\text { Índice Compuesto de Competitividad } \\
\text { Relativa (ICRp) }\end{array}$ & Santiago Ibarra Mirón & Tesis Doctoral & 2003 \\
\hline $\begin{array}{l}\text { Índice de Excelencia Competitiva de la } \\
\text { Unidad Estratégica de Fabricación } \\
(\text { IECuef) }\end{array}$ & Santiago Ibarra Mirón & Tesis Doctoral & 2003 \\
\hline $\begin{array}{l}\text { Índice de Diseño del Sistema de Control } \\
\text { de Gestión }(I N D d)\end{array}$ & Marisol Pérez Campana & Tesis doctoral. & 2005 \\
\hline $\begin{array}{l}\text { Índice Global de Desarrollo Sostenible } \\
(I G S)\end{array}$ & Luis Márquez Ortiz & Tesis Doctoral & 2007 \\
\hline $\begin{array}{l}\text { Índice de Actitud Estratégica en la } \\
\text { Transferencia de Tecnología (0-1) } \\
(I A E T T)\end{array}$ & Morayma Cazull Imbert & Tesis Doctoral & 2008 \\
\hline $\begin{array}{l}\text { Eficiencia Económica del Proceso de } \\
\text { Transferencia Tecnológica }(E E T T)\end{array}$ & Morayma Cazull Imbert & Tesis Doctoral & 2008 \\
\hline $\begin{array}{l}\text { Índice de Capacidad Gerencial del Pro- } \\
\text { ceso de Transferencia Tecnológica } \\
(\text { ICGPTT) }\end{array}$ & Morayma Cazull Imbert & Tesis Doctoral & 2008 \\
\hline
\end{tabular}




\begin{tabular}{|c|c|c|c|}
\hline Indicador Atractivo $(\boldsymbol{A T})$ & $\begin{array}{l}\text { Evis L. Diéguez Mate- } \\
\text { llán }\end{array}$ & Tesis doctoral. & 2008 \\
\hline $\begin{array}{l}\text { Indicador de Acercamiento al Cliente } \\
(I A C)\end{array}$ & $\begin{array}{l}\text { Evis L. Diéguez Mate- } \\
\text { llán }\end{array}$ & Tesis doctoral. & 2008 \\
\hline $\begin{array}{l}\text { Índice Integral de Desempeño de los } \\
\text { Procesos Hospitalarios }\end{array}$ & $\begin{array}{l}\text { Arialys Hernández Na- } \\
\text { riño }\end{array}$ & Tesis Doctoral & 2010 \\
\hline Índice Actuación Responsable (IAR) & José I. González Antuña & Tesis Doctoral & 2011 \\
\hline Índice de Valoración Ergonómica. & $\begin{array}{l}\text { Grether Lucía Real Pé- } \\
\text { rez. }\end{array}$ & $\begin{array}{l}\text { Pre defensa Tesis Doc- } \\
\text { toral }\end{array}$ & 2011 \\
\hline
\end{tabular}

Técnicas para la recopilación de los síntomas.

Algunas de las técnicas que se pueden utilizar para la recopilación inicial de la problemática existente son:

- Las entrevistas.

- La observación.

- La encuesta.

- La revisión de documentos.

- Método Delphi.

- Métodos multiatributo y multicriterio.

A continuación se describen las técnicas relacionadas anteriormente:

\section{Las entrevistas}

La entrevista es una conversación entre dos o más personas, en la cual una de ellas es el que pregunta (entrevistador). Es la comunicación interpersonal establecida entre investigador y el sujeto de estudio a fin de obtener respuestas verbales a los interrogantes planteados sobre el tema propuesto.

En ella, las personas dialogan con arreglo a ciertos esquemas o pautas de un problema o cuestión determinado, tiene un propósito profesional. Presupone la posibilidad de interacción verbal dentro de un proceso de acción recíproca. Como técnica de recolección va desde la interrogación estandarizada hasta la conversación libre, en ambos casos se recurre a una guía que puede ser un formulario o esquema de cuestiones que orientan la conversación. 
Existen diferentes tipos de entrevistas, (Beerli Palacios; 2000) las cuales se relacionan a continuación:

- En la entrevista estructurada, las preguntas están prefijadas y definidas con antelación e incluso, se le dan al entrevistado con anterioridad en forma de varias opciones y las respuestas son esperadas. Esta situación provoca una limitada libertad para formular preguntas independientes generadas por la interacción personal.

- Con la entrevista semiestructurada, se planifican previamente las preguntas pero con cierta libertad, para posteriormente abordar temas que puedan surgir en el desarrollo de la misma. Esta entrevista es más flexible y abierta, aunque los objetivos de la investigación rigen las preguntas del investigador, con base en el problema, los objetivos y las variables.

- La entrevista libre supone que no se estructuren ni planifiquen previamente las preguntas. Es la más ágil y proporciona más información en general, pero requiere cierto dominio por parte del entrevistador.

- La entrevista individual o a profundidad, es mantenida entre un entrevistador y un entrevistado para obtener que este último transmita oralmente su definición personal de la situación. Esta entrevista específicamente va dirigida a situaciones o individuo concretos, caracterizados y señalados previamente por haber tomado parte de la situación o poseer una experiencia definida.

- La entrevista focalizada, es mantenida de forma grupal, ofrece oportunidades de conocimiento y de análisis superiores. La experiencia en grupo promueve un ambiente en el cual se intercambian puntos de vista, los individuos encuentran una mayor facilidad de reflexión sobre el tema tratado.

- Por su parte, la entrevista de tensión pretende conocer el comportamiento del entrevistado en una situación en la cual es difícil mantener el control de sí mismo. Las situaciones se crean artificialmente, y se alternan situaciones amistosas y hostiles. Se discuten y critican las aportaciones del entrevistado, tratando de confundirle.

En síntesis, la entrevista es una forma de interacción social, donde el investigador se sitúa frente al investigado y le formula preguntas. Como instrumento de la investigación social, tiene una gran importancia pues permite obtener determinadas conclusiones sobre el tema investigado. 
Entre sus ventajas se encuentran:

- es una técnica eficaz para obtener datos relevantes y significativos desde el punto de vista de las ciencias sociales, con condición oral y verbal,

- es extremadamente flexible, capaz de adaptarse a cualquier condición, situación, personas, permitiendo la posibilidad de aclarar preguntas, orientar la investigación y resolver las dificultades que puede encontrar la persona entrevistada,

- la información que el entrevistador obtiene es muy superior que cuando se limita a la lectura de respuesta escrita, y

- a través de ella se pueden captar los gestos, los tonos de voz, los énfasis, los cuales aportan una importante información sobre el tema y las personas entrevistadas.

\section{La Observación.}

Requisitos de la observación como método científico:

- Objetividad: Debe entenderse como la correspondencia verdadera entre las observaciones y el objeto observado. El problema de la objetividad abarca tanto la validez de los resultados obtenidos como su generalización. De no existir, en la práctica, ocasiona una cadena de errores no controlables y que finalmente conducen a conclusiones falsas.

- La no causalidad: Surge como una necesidad de evitar que los resultados casuales sean tomados como resultados permanentes de las propiedades del sujeto o situación observada. La no causalidad se refiere entonces a la relación lógica entre el número, contenido y orden de las observaciones, en atención a las características del objeto estudiado y a los objetivos de la investigación.

- Repetitividad: Medida en que los datos obtenidos a través de la observación son permanentes y posibles de obtener, nuevamente en condiciones similares. En la práctica se cumple este requisito cuando se realizan observaciones en condiciones naturales, es decir, donde el observador no provoca o introduce variaciones y se limita sólo a observar y registrar los hechos tal como aparecen.

- Utilización de medios especiales para el registro de los hechos observados: Aquí se destacan especialmente: las guías o protocolos de observación 
(en las cuales se resume el conjunto de características del método), la cinematografía, la fotografía, la grabación de sonidos, el microscopio, etcétera.

Función fundamental de la observación:

- Asegurar aquella información empírica necesaria para plantear nuevos problemas y promover hipótesis, así como para su ulterior comprobación.

- Comprobar aquellas hipótesis o teorías que no puedan comprobarse con la ayuda del experimento. Las observaciones que van acompañadas de mediciones exactas, los resultados de tal comprobación pueden no ser peores que los del experimento, cosa que se confirma a través de toda la historia del desarrollo de la astronomía.

- La tercera función de la observación consiste en que en sus términos, se realiza la confrontación de los resultados obtenidos en el transcurso de la investigación teórica y se comprueba la adecuación y veracidad de éstos. Como no es posible confrontar directamente las bases abstractas de la teoría con la experiencia, hay que acudir a diversos procedimiento auxiliares, entre los cuales un papel muy importante lo desempeña la formulación de los resultados empíricos en los términos de la observación y en el lenguaje "observativo".

\section{La Encuesta}

La encuesta es una de las herramientas cuantitativas más utilizadas a la hora de obtener información primaria, pero ello no significa que sea adecuado recurrir siempre a ellas. Antes de hacer una encuesta hay que asegurarse de que realmente esa encuesta es necesaria, habrá que comprobar también que esté totalmente agotada la información secundaria sobre el tema que se va a estudiar.

También, se ha de verificar o demostrar que la información que se necesita no se puede obtener a través de técnicas cualitativas, ya que éstas son más baratas que las cuantitativas. Habrá también que estudiar o analizar el costo de la encuesta y los recursos disponibles para realizarla. También habrá que demostrar que el tema es de interés para la empresa y que, ciertamente, existe información disponible y accesible. Una vez que se comprueba la necesidad de la encuesta, el segundo paso es diseñar el plan de sondeo, es decir, desarrollar la encuesta, Beerli Palacios (2000). 
Existen tres tipos de encuestas: Encuestas por correo, Encuestas por teléfono, y Encuestas personales.

La encuesta de tipo personal se caracteriza por su, Alto índice de respuesta; Se conoce quien contesta; Evita la influencia de otras personas; Se reducen las respuestas evasivas; Utilización de material auxiliar; Obtención de datos secundarios por simple observación.

Etapas de una encuesta:

1. Definir la población a estudiar o el universo de estudio.

2. El universo o población a estudiar es el agregado de todos los elementos definidos antes de la selección de la muestra. Una población correctamente designada se debe definir en tres términos, la unidad de la muestra que es la unidad acerca de la cual se solicita información, el ámbito y el tiempo que hacen referencia al lugar y fecha de obtención de la información.

3. Determinar la unidad de la muestra que pueden ser individuos, familias, empresas, etcétera.

4. Definir de la muestra determinada, el elemento o elementos que se encuentran disponibles para su selección en alguna etapa del proceso de muestreo.

5. Determinar el tamaño de muestra representativo. Está condicionado al procedimiento de muestreo que se vaya a escoger.

La muestra es una parte del Universo debidamente elegida, que se somete a investigación científica en representación del conjunto del Universo.

Existen cuatro condiciones fundamentales que deben cumplir las muestras: comprender parte del Universo y no la totalidad de este, que su amplitud sea estadísticamente proporcionada a la magnitud del Universo, que no existan distorsiones en la elección de los elementos de la muestra, que sea representativa del Universo de forma tal que reproduzca sus características básicas en función de la investigación.

Para delimitar el tamaño de la muestra es necesario tener presente tres elementos básicos que se interrelacionan entre sí:

- El error de muestreo que viene dado por la desviación típica del estimador. 
- El error absoluto que se comete en la estimación como consecuencia de la técnica de muestreo: Es el margen de error que se está dispuesto a asumir en la estimación y se expresa en las mismas unidades que la variable a estimar.

- El coeficiente o intervalo de confianza que se utiliza en la estimación: Indica la probabilidad de que se cumpla que efectivamente la diferencia entre la estimación y el valor real este comprendido en los márgenes del error absoluto.

Pk $=$ Probabilidad $[|x-X| \leq e]$

Para un coeficiente de confianza(Pk)de:68.26\%le corresponde un intervalo de confianza de1x, desviación típica $(K=1)$, para 95.46\%, 2xdesviación típica $(K=2)$ y si $99.73 \% \%$ entonces $3 x$ desviación típica $(K=3)$. Se recomienda el uso de $\mathrm{K}=2$, donde la probabilidad es de $95.46 \%$.

El error absoluto esK veces el error de muestreo $(\mathrm{e}=\mathrm{K} \times$ desviación típica $)$.

Determinación del tamaño de muestra:

Tabla 2. Fórmulas para la determinación del tamaño de muestra. (Beerli Palacios, 2000)

\begin{tabular}{ll}
\hline Tamaño de la muestra para poblaciones infinitas. & $n=\frac{k^{2} P Q}{e^{2}}$ \\
\hline Tamaño de la muestra para poblaciones finitas & $n=\frac{N K^{2} P Q}{e^{2}(N-1)+k^{2} P Q}$ \\
Tamaño de la muestra para la estimación de totales. & $n=\frac{K^{2}+N^{2} S^{2}}{e^{2}+K^{2} N S^{2}}$ \\
Tamaño de la muestra para la estimación de medias. & $n=\frac{K^{2} N S^{2}}{N e^{2}+K^{2} S^{2}}$ \\
\hline
\end{tabular}

Dónde:

n: $\quad$ Tamaño de la muestra.

K: $\quad$ Error absoluto/error de muestreo. 
N: Tamaño de la población.

P: $\quad$ Probabilidad que se produzca un fenómeno o proporción de la muestra.

$Q: \quad$ La probabilidad contraria a que se produzca $P$.

e: $\quad$ Error absoluto.

Nota: Una población es considerada infinita cuando excede los 10.000 individuos.

Selección del método o procedimiento de muestreo

Se puede utilizar métodos probabilísticos, semiprobabilísticos y no probabilísticos:

- Probabilístico:

- Aleatorio Simple.

- Aleatorio Sistemático.

○ Aleatorio Estratificado.

- Por Conglomerados.

- Semiprobabilístico:

- Por rutas Aleatorias.

- No probabilístico.

- Razonado.

- Por Juicios o discrecional.

○ Por cuotas.

○ Bola de Nieve.

6. Diseñar y preparar el cuestionario. En esta fase habrá que determinar el contenido y formato del cuestionario.

Para el diseño del cuestionario hay que tener en cuenta el contenido de las preguntas, el tipo de pregunta que se va a realizar y la secuencia de las mismas. Es importante realizar un pretest y revisarlo y previo a la elaboración final del cuestionario.

7. Organización y realización del trabajo de campo:

Información a todos los elementos pertenecientes a la población a estudiar de los objetivos del estudio.

8. Creación de la base de datos y análisis de la información: 
Se recogen todas las respuestas a la encuestas y se montan en una base de datos para su procesamiento.

9. Interpretación de los resultados e informe final:

Se interpretan los resultados extraídos de las respuestas y se realiza el informe final del resultado de la aplicación de la encuesta.

\section{Revisión de documentos}

Esta técnica es muy usada por la facilidad de recogida de la información. Consiste en la revisión de documentos asociados a la investigación que se pretende efectuar.

Comúnmente, esta revisión documental para el caso del Control de Gestión abarca los aspectos siguientes:

- Revisión de documentos asociados al proceso de planificación y Control de la empresa (Planificación Estratégica, Cuadro de Mando Integral u otros documentos asociados a los procesos de Planificación y Control empresarial),

- Revisión de documentos normativos o de referencias para el trabajo empresarial (Perfeccionamiento Empresarial, Resolución 297, Normas ISO, etc),

- Teoría científica publicada acerca del tema tratado,

- Buenas prácticas existentes en el mundo empresarial (Benchmarking).

\section{Método Delphi}

Es uno de los más conocidos y consiste en seleccionar una serie de expertos (que entre sí desconocen el hecho de haber sido seleccionados, dirigidos por un coordinador que actúa de intermediario entre ellos. Este les solicita opiniones y previsiones, y de entre las recibidas selecciona aquellas que caen en el 25\%de las más pesimistas y el $25 \%$ de las más optimistas. Se le solicita explicaciones a esas personas de cómo justifican ellos este hecho. Toda la información recibida, incluidas las justificaciones, son enviadas a los expertos y se repite el proceso hasta que se arribe a un consenso (Medina león et al. 2002).

\section{Métodos multiatributo y multicriterio}

Las llamadas técnicas para la toma de decisión multicriterio se suelen dividir en dos grandes bloques, según las características de los problemas que afronten: 
- Múltiples objetivos (MODM): Aquellos problemas donde el conjunto de soluciones es infinito.

- Múltiples atributos (MADM): Aquellos problemas donde el conjunto de soluciones es finito.

- Los modelos que representan los problemas multiobjetivos atendiendo al enfoque con que proceden pueden ser:

- Modelos de programación de metas.

- Método del vector máximo.

- Métodos iterativos.

- En los problemas multiatributos para seleccionar la mejor alternativa existen dos enfoques:

- Normativo (escuela inglesa): Método de los pesos aditivos.

- Descriptivos (escuela francesa): Métodos de sobre-clasificación, los ELECTRE.

\section{Reducción del listado.}

Una vez obtenida la información primaria para la obtención del índice, se hace necesario la reducción del listado con la intención de convertirlo en número racional y manejable de información para la gestión. Entre las formas comúnmente usadas con este propósito se encuentran:

- Reducción del listado según el procede del procedimiento Brainstorming.

- Método Kendall.

- Combinación de los anteriores (reducción a una cifra manejable y luego Kendall).

- Método Torgerson.

\section{Procedimiento Brainstorming}

El método de la tormenta de Ideas o tormenta cerebral se le atribuye a Osborn, quién en 1938 lo utilizó para reflexiones en grupo. Para su utilización se requiere de un grupo de participantes capaces de aportar soluciones al problema que se estudia, guiados y estimulados eficazmente por un facilitador y auxiliado por uno o más registradores. El facilitador puede ser el jefe del grupo, un miembro cualquiera o una persona externa. 
Su aporte más importante es el de separar la generación de ideas de su evaluación y crítica, pues esta última es capaz de matar las ideas antes de que lleguen a expresarse o desarrollarse.

La ejecución de la tormenta de ideas debe realizarse sobre la base de las siguientes reglas o principios (Medina León et al. 2002):

- Es una generación de ideas en grupo.

- Se suprime toda crítica.

- Toda idea por ridícula o absurda que parezca, debe manifestarse en la reunión, sólo será absurda realmente las ideas que no se manifiesten. Se admiten ideas contrarias, mejoradas, complementarias e incluso iguales.

- La cantidad de ideas es la base del método, no la calidad de las mismas. Cuántas más ideas sean generadas, más posible será llegar a buenos resultados y alcanzar entonces la calidad deseada. Fomentar ideas en bruto e imponer un trabajo posterior de análisis y selección que también se puede hacer en grupo.

- Se estimula la generación de nuevas ideas y se mejoran y transforman las emitidas por los demás.

- No debe utilizarse para soluciones claras o que podamos encontrar basándonos en la experiencia, tampoco para problemas que admitan una sola solución. Se deberá abordar un tema preciso.

- Los criterios acerca del tiempo de duración máxima de la sesión varían acorde a los distintos autores entre 15 minutos y 2 horas.

- Respecto al número de participantes: Companys Pascual (1989) plantea entre 7 ó 8, otros autores entre 6 y 40. Ciertamente, en la medida que el número sea mayor y más cercano a 40 será más difícil dirigir al grupo.

- Debe velarse por la calidad de los participantes, los que deben conocer del tema, poseer formaciones y edades diferentes, resultando factible y útil su renovación sistemática.

- El Brainstorming y otros métodos similares resultan de notable ayuda en las primeras etapas de la previsión tecnológica y resultan el origen de los escenarios, siendo la fuente de ideas que pueden ser desarrolladas en el futuro.

- El facilitador o coordinador puede y debe estimular la sesión mediante: la búsqueda de analogías, asociaciones de palabras y juegos, listas de preguntas 
o de palabras, etc. En todo momento deberá tener el control de la sesión y garantizar que todas las ideas sean registradas tal y como han sido emitidas, siendo útil para esto repetir la idea una vez planteada y cerciorarse de que fue registrada. Deberá también estimular al enriquecimiento, modificación o mejoramiento de las ideas planteadas.

- La principal dificultad de la utilización del Brainstorming estriba en la ordenación de las ideas, su reducción a un número manejable que puedan presentarse a las personas que deberán seleccionarlas y eventualmente utilizarlas. Pueden utilizarse diversos procedimientos pero la actitud del facilitador es esencial.

- Toda idea escrita pasa a ser del grupo.

- Se considera positivo un ambiente informal.

La recopilación de estas ideas puede ser realizada también de forma escrita, lo que garantiza un mayor anonimato de los participantes y recomendable cuando el jefe del grupo está presente y posee alta influencia sobre sus subordinados. Su aplicación garantiza un nivel de elaboración mayor de las ideas; pero no logra un ambiente tan informal. En todas las variantes se comienza por el planteamiento del problema y la requerida motivación de los participantes que proceden a escribir sus ideas, durante un tiempo prefijado, en pliegos de papel. Las variantes más conocidas son:

- Embalse de ideas: Cada participante coloca papel en un recipiente, de donde puede también extraer otra hoja la que puede enriquecer con nuevas ideas.

- Exposición de ideas: Las tarjetas creadas por los participantes se clavan en la pizarra y los distintos miembros pueden aportar otras nuevas en las tarjetas estimulados por su lectura.

- Grupos nominales: Los miembros del grupo colocan sus ideas en las tarjetas y se las entregan al facilitador que procede a registrar estas ideas en la memoria de grupo procurando no seguir un orden predeterminado en la lectura de las tarjetas. El facilitador puede recoger las tarjetas y repartirlas nuevamente en un orden contrario al que fueron recogidas y se permite un nuevo tiempo para generar nuevas ideas antes de pasar a crear la memoria del grupo.

- 6-3-5: Participan seis personas, que generan tres ideas cada uno en cada oportunidad y poseen cinco minutos para pensar en sus tres posibles ideas. Pasados los cinco minutos el facilitador ordena pasar su tarjeta al miembro que 
esté a su derecha repitiendo el procedimiento seis veces. Pueden obtenerse hasta 108 ideas en 30 minutos.

\section{Método Kendall}

Este método permite verificar la concordancia entre los juicios expresados por el grupo de expertos con respecto a las evaluaciones y reducir el listado. El algoritmo a seguir consiste en solicitar a cada experto su criterio acerca del ordenamiento según el grado de importancia de cada una de los síntomas o posibles elementos del indicador. Con estos se conforma la tabla 3.

Dónde:

$A_{i j}: \quad$ Ponderación sobre el criterio de la característica o variable $i$, según el experto $j$.

K: $\quad$ Cantidad de características o número de índices a evaluar.

m: $\quad$ Número de expertos que emiten criterio.

T: $\quad$ Factor de concordancia.

$W: \quad$ Coeficiente de concordancia o Kendall.

Tabla 3. Ejemplo de Aplicación del Coeficiente de Kendall para la selección de los procesos relevantes.

\begin{tabular}{|c|c|c|c|c|c|c|c|c|c|c|c|c|}
\hline \multirow[t]{2}{*}{$K$} & \multirow[t]{2}{*}{ Procesos } & \multicolumn{8}{|c|}{$\operatorname{Expertos}(\boldsymbol{m})$} & \multirow[b]{2}{*}{$\Delta$} & \multirow[b]{2}{*}{$\Delta^{2}$} & \multirow[b]{2}{*}{ Selección } \\
\hline & & E1 & E2 & E3 & E4 & E5 & E6 & E7 & $\sum A_{i}$ & & & \\
\hline 1 & & $A_{i j}$ & $A_{i j}$ & $A_{i j}$ & $A_{i j}$ & $A_{i j}$ & $A_{i j}$ & $A_{i j}$ & & & & \\
\hline 2 & & $A_{i j}$ & $A_{i j}$ & $A_{i j}$ & $A_{i j}$ & $A_{i j}$ & $A_{i j}$ & $A_{i j}$ & & & & \\
\hline 3 & & $A_{i j}$ & $A_{i j}$ & $A_{i j}$ & $A_{i j}$ & $A_{i j}$ & $A_{i j}$ & $A_{i j}$ & & & & \\
\hline$\ldots$ & & $A_{i j}$ & $A_{i j}$ & $A_{i j}$ & $A_{i j}$ & $A_{i j}$ & $A_{i j}$ & $A_{i j}$ & & & & \\
\hline$n$ & & $A_{i j}$ & $A_{i j}$ & $A_{i j}$ & $A_{i j}$ & $A_{i j}$ & $A_{i j}$ & $A_{i j}$ & & & & \\
\hline & & & & & & & & $\sum A_{i}$ & & $\sum \Delta^{2}$ & & \\
\hline
\end{tabular}

Para un mejor procesamiento es necesario obtener la suma de los criterios de los expertos sobre las características $i$, de la forma siguiente: 


$$
\begin{aligned}
& \sum_{i=1}^{m} A_{i} \\
& \sum_{j=1}^{k} \sum_{i=1}^{m} A_{i j}
\end{aligned}
$$

Se halla el factor de concordancia $(T)$ a través de la fórmula siguiente:

$T=\frac{1}{K}\left(\sum_{j=1}^{k} \sum_{i=1}^{m} A_{i j}\right)$

$\sum_{i=1}^{m} A_{i} \leq T$

Para comprobar la concordancia entre los expertos se calcula el coeficiente de concordancia o Kendall $(W)$ :

$W=\frac{12 \sum \Delta^{2}}{m^{2}\left(k^{3}-k\right)}$

$\Delta=\left(\sum_{i=1}^{m} A_{i}-T\right)$

$\Delta^{2}=\sum_{i=1}^{m}\left(A_{i}-T\right)^{2}$

Si $W \geq 0.5$ se puede decir que existe concordancia de criterios entre todos los miembros que conforman el panel de experto, por lo que el estudio realizado es confiable.

\section{Modelo Torgerson}

Existen distintas técnicas y modelos para la valoración de consensos para los expertos, no obstante este se considera útil cuando las escalas empleadas en los instrumentos aplicados a los expertos sean ordinales. El Modelo Matemático Torgerson, que es una variante del método Thurstone (Piloto Fleitas, 2011).

Con el modelo Torgerson se trata de dar objetividad a los criterios de los expertos u otro personal encuestado, al convertir la escala ordinal en escala de intervalo (de cualitativo a cuantitativo). 
Para lograr la objetividad a partir de los criterios subjetivos de los expertos el modelo se apoya en dos leyes de la psicología social:

- Ley de los juicios comparativos.

- Ley de los juicios categóricos.

La primera de estas leyes, expresa que la frecuencia de las preferencias de un indicador ante otro es una función de la intensidad con que ambos poseen la característica considerada. La segunda, se refiere a que las frecuencias de preferencia de un indicador poseen una característica a escalar, que refleja la intensidad con que posee la característica considerada. De esta forma, se refleja el hecho de que la coincidencia de criterios subjetivos permite la elaboración de una escala que posee ciertas condiciones de objetividad. Ambas leyes se fundamentan en expresiones matemáticas no abordadas en el presente trabajo.

Este Modelo se sustenta en los presupuestos siguientes:

1. Cada objeto (indicador) se corresponde con la dimensión subjetiva de una variable aleatoria distribuida normalmente, cuya media, $m$, es el valor de escala de ese objeto. Todas las varianzas son iguales.

2. Cada límite de categoría se corresponde con la dimensión subjetiva de una variable aleatoria distribuida normalmente, cuya media, $t$, es el valor de escala de ese límite. Todas las variables son iguales.

3. Las variables aleatorias que representan tanto a los objetos como a los límites, son independientes. Una variable no puede contener valores de otra.

4. Regla de decisión: un objeto a, pertenece a la $k$ - ésimacategoría cuando su valor de escala $x$, está entre los valores de los límites de orden $k-1$ y $k$. Esto regla deja bien definida la frontera entre cada una de las categorías asumidas para los indicadores. Piloto Fleitas, (2011).

Este modelo permite convertir los juicios ordinales, emitidos por expertos independientes, acerca de los indicadores, en un instrumento que expresa su posición relativa en un continuo; o sea, permite llevar las escalas ordinales a escala de intervalo (números reales) y de esta forma conocer los límites, en valores reales, en que se encuentra cada categoría; por ejemplo:

Excelente, MuyBien, ...; o de: 5, 4, 3,... 
Metodología para aplicar el Modelo Matemático Torgerson

1. Operacionalización de las variables a emplear.

Es importante aclarar que cuando se solicite información a los expertos acerca de estimar la posible categorización de cada uno de los indicadores o variables propuestas, es necesario que el investigador deje bien definidos los conceptos que asume para cada variable, así como establecer su operacionalización, que no es más que disponer los distintos parámetros e indicadores que permitan medir la variable en el sujeto, para posibilitar una mejor comprensión y evaluación de los juicios emitidos por los expertos.

2. Compilación de la frecuencia absoluta.

3. Determinación de la frecuencia acumulada.

4. Determinación de la frecuencia relativa acumulada.

5. Cálculo de los puntos de cortes y escala de los indicadores.

5.1. Determinación de los valores normales estándar inversos.

- Utilización de las Tablas de la distribución normal.

- Utilizando paquetes informáticos con esta función como el Microsoft Excel.

5.2. Búsqueda de los puntos de corte.

Una vez determinada cada imagen por la Curva Normal Estándar Inversa, se suma cada columna y cada fila y se promedian.

A los valores que resultan de las operaciones anteriores se les llama puntos de corte, y determinan los valores del intervalo en que van a estar comprendidas las variables cualitativas.

\subsection{Determinación del valor $N$.}

Se promedia los valores de los puntos de cortes, que no son más que los promedios de cada categoría (columna), por lo que se obtiene el promedio de promedio, llamado valor límite $N$.

\subsection{Determinación del rango en el que se encuentra la variable.}

Para saber en qué rango verdaderamente está la variable, se le resta al valor límite $N$ el valor promedio de cada fila $P$, el valor obtenido es comparado con el punto de corte o límite superior de cada categoría. 
6. Determinación del nivel de consenso.

El nivel de consenso $(C)$ se determina por la expresión:

$$
c=\left(1-\frac{V_{n}}{V_{t}}\right)
$$

Dónde:

C: $\quad$ coeficiente de concordancia.

$V_{n}: \quad$ Votos negativos.

$V_{t}: \quad$ Votos totales.

Regla de decisión: Sí $C>75 \%$, se considera que hay consenso.

En caso de que no haya consenso entre los expertos $C<75 \%$, se deberá hacer otra vuelta de consulta, realizándose las modificaciones y aclaraciones necesarias que permitan obtener consenso a las nuevas modificaciones y adecuaciones de la nueva propuesta de estos expertos.

\section{Determinación de coeficientes de prioridades (pesos).}

Cuétara Sánchez (2009) recomienda diferentes métodos factibles de ser aplicados a la situación que se estudia, entre ellos:

- Método Kendall.

- Triángulo de Fuller.

- Método de las jerarquías analíticas (AHP).

- Programación multiobjetivo.

\section{Triángulo de Fuller}

El método de comparación por parejas mediante o Triángulo de Füller, requiere sólo de la opinión de un decidor o experto.

Los criterios son codificados con números naturales $(1,2, \ldots, K)$. Se elabora un triángulo que servirá de base de comparación al experto, cuyas filas están formadas por un arreglo de pares de números naturales, como siguen: 


\section{\begin{tabular}{lllll}
1 & 1 & $\ldots$ & 1 & $n-1$ \\
2 & 3 & $\ldots$ & $n$ & \\
\hline
\end{tabular}}

\begin{tabular}{llll}
2 & $\ldots$ & 2 & $n-2$ \\
3 & $\ldots$ & $n$ & \\
\hline
\end{tabular}

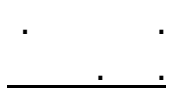

$\begin{array}{cc}n-1 & 1 \\ n & \end{array}$

El experto es invitado a seleccionar en cada par, la característica más importante, la se marca con un asterisco y se contabiliza la cantidad de que cada fila (criterio) fue seleccionada como la más significativa $\left(\mu_{i}\right)$.

La cantidad de comparaciones posibles, o el total de comparaciones se obtiene por la expresión siguiente:

$N=\frac{K(K-1)}{2}$

Dónde:

$N: \quad$ Total de comparaciones realizadas por el experto.

$K: \quad$ Número de características o variables.

Los pesos de los criterios se denotan por $V_{i}$ y se obtienen con la utilización la expresión siguiente:

$V_{i}=\frac{m_{i}}{N}$

Dónde:

$\mu_{i}: \quad$ Cantidad de veces que se marca la característica $i$.

$i: \quad 1,2, \ldots, K$.

Las características o variables de mayor peso, constituyen las de mayor importancia y el valor de cada una representa su peso relativo. 


\section{Método Saaty}

A finales de la década de los 70, Thomas Saaty introdujo un método multicriterio discreto conocido por AHP (en inglés Analytical Hierarchy Process) de gran impacto tanto a nivel teórico como aplicado.

Se supone que el problema a decidir consiste en elegir el trazado de un tramo de autopista. Existen tres trazados posibles (las elecciones o alternativas, que denominaremos $A, B y C)$, que se evalúan en base a tres criterios relevantes: costo de ejecución, impacto ambiental y tiempo de ejecución. Es decir, enfrentamos un método multicriterio discreto $3 \times 3$ (3 alternativas y 3 criterios).

La estructura jerárquica del problema dentro del enfoque de Saaty queda representada en la Tabla 4. El primer nivel o jerarquía de la estructura corresponde al propósito del problema, el segundo a los criterios y el tercero a las alternativas o elecciones posibles.

Tabla 4: Trazados alternativos de una autopista: Representación Jerárquica (Romero, 1996).

\begin{tabular}{ccccc}
\hline Nivel 1 & Propósito & & \multicolumn{2}{c}{ Elección de un trazado de autopista } \\
\hline Nivel 2 & Criterios & Costo & Impacto ambiental & Tiempo de ejecución. \\
Nivel 3 & Alternativas & A & B & C \\
\hline
\end{tabular}

Una vez conceptualizada la estructura jerárquica del problema, se establece una fuerte interacción con el centro decisor para que emita sus juicios de valor o preferencias en cada uno de los niveles jerárquicos establecidos. Esta tarea consiste en una comparación de valores subjetivos "por parejas"; es decir, el centro decisor tiene que emitir $n(n-1) / 2$ juicios de valor $(3(3-1) / 2=3)$ (para el caso que se expone) sobre la importancia relativa de criterios y alternativas.

Aunque no siempre es necesario en muchos métodos multicriterio, resulta esencial proceder a la normalización de los diferentes criterios en consideración. La normalización es necesaria, al menos por tres razones:

- en la mayor parte de los contextos que requieren de decisiones las unidades en que están medidos los diferentes criterios suelen ser muy diferentes, 
- los valores alcanzables por los diferentes criterios pueden ser muy diferentes,

- más facilidad en las tareas comparativas entre criterios por el centro decisor, con el propósito de obtener indicadores de sus preferencias.

Los valores numéricos que propone aplicar Saaty son los siguientes:

(1) cuando los criterios son de la misma importancia

(3) moderada importancia de un criterio con respecto a otro

(5) fuerte importancia

(7) demostrada importancia

(9) extrema importancia

Asimismo, Saaty sugiere valores intermedios para juicios de valor contiguos.

Es interesante observar que, por su propia construcción, este tipo de matrices a lo "Saaty" poseen propiedades recíprocas (esto es, $a_{i j}=1 / a_{j i}$ ). A partir de la matriz anterior se pretende encontrar un vector de pesos $\left(W_{1}, W_{2}, W_{3}, W_{4}\right)$ que resulte consistente con las preferencias subjetivas mostradas por el centro decisor y reflejadas en la comentada matriz.

Para el nivel jerárquico segundo, los valores subjetivos que se suponen ha emitido el centro decisor para los datos de nuestro ejemplo quedan reflejados en la matriz de la Tabla 5.

Tabla 5: Valores emitidos por el centro decisor.

\begin{tabular}{lccc}
\hline & Costo & Impacto ambiental & Tiempo de ejecución \\
\hline Costo & 1 & 2 & 5 \\
Impacto ambiental & $1 / 2$ & 1 & 3 \\
Tiempo de ejecución & $1 / 5$ & $1 / 3$ & 1 \\
\hline
\end{tabular}

Es interesante observar que para aplicar el método AHP no hace falta información cuantitativa acerca del resultado que alcanza cada alternativa en cada uno de los criterios considerados, sino tan solo los juicios de valor del centro decisor. 
El paso siguiente es la aplicación del método AHP consiste en obtener un sistema de pesos que resulte consistente con las preferencias subjetivas mostradas por el centro decisor y recogidas en la matriz de comparación "por parejas" de la Tabla 5. Para ello se tendrá que encontrar un conjunto de valores $W$ no negativos que satisfaga las tres ecuaciones que derivan de la matriz:

$W_{1}-2 W_{2}=0$

$W_{1}-5 W_{3}=0$

$W_{2}-3 W_{3}=0$

Las lógicas y comprensibles inconsistencias de los juicios de valor del centro decisor hacen, como es habitual, que la única solución que admite el anterior sistema de ecuaciones sea la trivial $W_{1}=W_{2}=W_{3}=0$. Por tanto, debemos de encontrar el conjunto de pesos W que más se aproxime a los verdaderos. Para abordar esta tarea podemos recurrir a diferentes procedimientos matemáticos como el cálculo de medias geométricas, el método del autovalor máximo sugerido por Saaty, o a la formulación de un modelo de programación por metas ponderadas.

El vector $\left[W_{1}=0,588 ; W_{2}=0,294 ; W_{3}=0,118\right]$ representa la estimación de pesos obtenida y el valor en el óptimo de la función objetivo (i. e. 0,06 ) el ratio de consistencia mostrado por el centro decisor al manifestar sus preferencias.

Una vez determinados los pesos para el nivel jerárquico 2, el paso siguiente en la aplicación del método AHP consiste en interaccionar nuevamente con el centro decisor, pero ahora en el nivel jerárquico 3. Para ello, el centro decisor tiene que mostrar sus juicios de valor cuando se confronta cada alternativa (trazado de la autopista) con cada criterio. Las tres matrices de comparación para el nivel jerárquico tres están recogidas en la Tabla 6.

Nuevamente, la interpretación de los elementos de las matrices de comparación es obvia. Así, para la primera matriz de comparación (p. ej. la que se refiere al coste) tenemos que, para el centro decisor, el trazado $A$ es seis veces preferido al $B y$ tres veces preferido al $C$ en términos del criterio costo, etc. Las tres matrices de comparación están ampliadas con una columna en la que figuran recogidas las estimaciones de los pesos que resultan consistentes con las preferencias mostradas por el centro decisor. 
Dichas estimaciones se obtiene de recurrir nuevamente a un modelo de programación por metas ponderadas.

Una vez obtenidos los estimadores de los pesos para los niveles jerárquicos 2 y 3 , el paso siguiente -y último- del método AHP consiste en obtener unos pesos globales para ambos niveles jerárquicos. Esta tarea se aborda por medio de una agregación multiplicativa entre niveles jerárquicos. Así, para el trazado A el peso global es: $0,667 \times 0,588+0,069 \times 0,294+0,143 \times 0,118=0,429$. En la Tabla 6 figuran recogidos tanto los pesos obtenidos en los niveles jerárquicos 2 y 3 como los pesos globales.

Tabla 6: Matriz de estimación por parejas y estimaciones de pesos relativos para el nivel jerárquico 3. Costo.

\begin{tabular}{ccccc}
\hline & A & B & C & Peso $\boldsymbol{W}$ \\
\hline A & 1 & 6 & 3 & 0.667 \\
B & $1 / 6$ & 1 & $1 / 2$ & 0.111 \\
\hline C & $1 / 3$ & 2 & 1 & 0.222 \\
\hline
\end{tabular}

Tabla 7: Matriz de estimación por parejas y estimaciones de pesos relativos para el nivel jerárquico 3. Tiempo de ejecución.

\begin{tabular}{ccccc}
\hline & A & B & C & Peso $\boldsymbol{W}$ \\
\hline A & 1 & $1 / 2$ & $1 / 4$ & 0.143 \\
B & 2 & 1 & $1 / 2$ & 0.28 \\
& & & & \\
C & 4 & 2 & 1 & 0.577 \\
\hline
\end{tabular}

\section{Programación multiobjetivo}

La Programación Multiobjetivo puede definirse como una parte de la Investigación Operativa que trata de proporcionar métodos útiles -eficientes- para la toma de decisiones sobre problemas que incluyen diversidad de objetivos, a veces contradictorios, que son evaluados de acuerdo a múltiples criterios y donde no es evidente la mejor u óptima alternativa. 


\section{Determinación del índice.}

La determinación de los Índices Integrales se realiza sobre la base del cálculo aritmético, usualmente una función aditiva; se requiere desarrollar una expresión del tipo como las que se muestran en la tabla 8 , en la que se realiza una comparación entre el máximo nivel que puede ser alcanzado (en el caso de que todos los indicadores obtengan la más alta puntuación) y el que posee la organización en la actualidad.

Tabla 8. Expresiones utilizada para la determinación de Índices Integrales (Piloto Fleitas, 2011)

\begin{tabular}{|c|c|c|}
\hline Nombre & Autor y año & Expresiones matemáticas \\
\hline $\begin{array}{l}\text { Índice Integral } \\
\text { de Eficiencia } \\
\text { Financiera } \\
\operatorname{ra}(I I E F H)\end{array}$ & $\begin{array}{l}\text { Nogueira Ri- } \\
\text { vera, } 2002\end{array}$ & $\begin{array}{l}I I E F H=\frac{\sum_{j=1}^{k} V_{j} \times P_{j}}{\sum_{j=1}^{k} V_{j}} \\
V_{j}: \text { Peso relativo de cada indicador (determinado por el método de } \\
\text { Kendall). } \\
P_{j}: \text { Comportamiento de cada indicador en la empresa analizada. } \\
k: \text { Cantidad de indicadores. }\end{array}$ \\
\hline $\begin{array}{l}\text { Índice de Exper- } \\
\operatorname{ticidad}(I E)\end{array}$ & $\begin{array}{l}\text { Oñate Ramos, } \\
2002\end{array}$ & $\begin{array}{l}I E_{j_{1}}=\sum_{j=1}^{n} W_{j} \times C_{j} \forall j_{1}=1 \ldots n \\
W: \text { Importancia que se le atribuye al } j \text { criterio para el cálculo de } I E . \\
C: \text { Valores normalizados de las } j \text { variables. } \\
n: \text { Total de expertos propuestos que se valoran. }\end{array}$ \\
\hline $\begin{array}{l}\text { Índice de Satis- } \\
\text { facción al Cliente } \\
(I S C)\end{array}$ & $\begin{array}{l}\text { Diéguez Mate- } \\
\text { llán, } 2008\end{array}$ & $\begin{array}{l}I S C=\sum_{i=1}^{n} W_{i} \times V_{i} \\
W: \text { Peso del atributo } i \text { dado por el cliente externo. } \\
V: \text { Valoración dada por el cliente } i \text { externo del atributo } i . \\
n: \text { Número de atributo del servicio. }\end{array}$ \\
\hline
\end{tabular}




\begin{tabular}{|c|c|c|}
\hline $\begin{array}{l}\text { Indicador Atrac- } \\
\text { tivo }(A T)\end{array}$ & $\begin{array}{l}\text { Diéguez Mate- } \\
\text { llán, } 2008\end{array}$ & $\begin{array}{l}A t_{i}=\sum_{j=1}^{n} A_{i j} \times W_{i j} \\
A_{i j}: \text { Media del grado de presencia percibida del atributo atractivo } \\
j \text { en el SC } i . \\
W_{i j}: \text { Peso del artículo de atractivo } j \text { en el SC } i . \\
A t_{i}: \text { Atractivo del SC } i . \\
I_{i j}: \text { Importancia media del atributo } j \text { en el SC } i . \\
i: \text { SC; desde } i=1 \ldots \ldots n_{\mathrm{y}} j: \mathrm{SC} ; \text { desde } j=1, \ldots, m .\end{array}$ \\
\hline $\begin{array}{l}\text { Excelencia de los } \\
\text { procesos hotele- } \\
\text { ros }(E P H)\end{array}$ & $\begin{array}{l}\text { Negrín Sosa, } \\
2003\end{array}$ & $\begin{array}{l}E P H=\sum_{i=1}^{11} P_{i} \times V_{i} \\
V_{i}: \text { Ponderación del medidor } i \text { respecto al total. } \\
P_{i}: \text { Puntuación otorgada del medidor } i \text { por los expertos evaluadores. }\end{array}$ \\
\hline $\begin{array}{l}\text { Nivel de Acer- } \\
\text { camiento a los } \\
\text { deseos de los } \\
\text { clientes que al- } \\
\text { canza una em- } \\
\text { presa m } \\
(N A D C m)\end{array}$ & $\begin{array}{l}\text { Sarache Cas- } \\
\text { tro, } 2003\end{array}$ & $\begin{array}{l}N A D C m=\left[\sum_{j=1}^{n} W_{j}\left(\sum_{i=1}^{m} W_{i j} \times C_{i j}\right)\right] \times R S m \\
W_{j} \text { :Peso del factor } i \text {. } \\
W_{i j}: \text { Peso del componente } i \text { en el factor } j \text {. } \\
C_{i j}: \text { Calificación obtenida por la empresa por el componente } i \text { del } \\
\text { factor } j \text {. } \\
R S m: \text { Grado de cumplimiento de la responsabilidad social de la } \\
\text { empresa } m \text {. }\end{array}$ \\
\hline
\end{tabular}

$\begin{array}{lll}\text { Intensidad inno- } & \text { Suárez Her- } & \text { IIEG }=\frac{\sum P_{i} \times W_{i}}{5 \times \sum W_{i}} \\ \text { vadora en la em- } & \text { nández, } & P_{i} \text { :Puntuación otorgada a la variable } i . \\ \text { presa ganadera } & 2003 & W_{i} \text { :Peso específico según el grado de importancia de la variable } i . \\ (\text { IIEG }) & \end{array}$

\begin{tabular}{|c|c|}
\hline $\begin{array}{l}\text { Nivel de excelen- } \\
\text { cia en empresas }\end{array}$ & $\begin{array}{l}\text { Suárez Her- } \\
\text { nández, }\end{array}$ \\
\hline $\begin{array}{l}\text { ganaderas } \\
(\boldsymbol{E} O \boldsymbol{E} \boldsymbol{G})\end{array}$ & 2003 \\
\hline
\end{tabular}

$E O E G=\left(\frac{\sum_{j=1}^{k} P_{j} \times V_{j}}{10 \times \sum_{i=1}^{k} V_{i}}\right) \times 100$

$P_{j}$ :Puntuación otorgada al indicador $i$.

$V_{i}$ :Peso relativo o ponderación del indicador $i$.

$k$ :Cantidad de indicadores. 


\begin{tabular}{|c|c|c|}
\hline $\begin{array}{l}\text { Nivel de excelen- } \\
\text { cia de la tecnolo- } \\
\text { gía en la empresa } \\
(\text { ETE) }\end{array}$ & $\begin{array}{l}\text { Suárez Her- } \\
\text { nández, } 2003\end{array}$ & $\begin{array}{l}E T E=\left(\frac{\sum P_{i} \times V_{i}}{\sum V_{i}}\right) \times 10 \\
P_{i}: \text { Puntuación asignada a cada índice. } \\
V_{i}: \text { Peso específico del índice } i .\end{array}$ \\
\hline $\begin{array}{l}\text { Índice de actitud } \\
\text { estratégica en la } \\
\text { transferencia de } \\
\text { tecnología (0-1) } \\
(\text { IAETT })\end{array}$ & $\begin{array}{l}\text { Cazull Imbert, } \\
2008\end{array}$ & $\begin{array}{l}I A E T T=\frac{1}{4} \times \sum_{j=1}^{m} P_{j} \times \sum_{i=1}^{n}\left(K_{i} \times P_{i}\right) \\
P_{j}: \text { Peso relativo del factor actitud estratégica en transferencia de } \\
\text { tecnología } 0 \leq P_{j} \geq 1 . \\
P_{i}: \text { Peso relativo de cada variable en el grupo } 0 \leq P i \geq 1 . \\
K_{i}: \text { Comportamiento de cada variable } i \text { en transferencia de tecnolo- } \\
\text { gía analizada } \\
n: \text { Cantidad de variable de la actitud estratégica. } \\
m: \text { Cantidad de grupo de factores. }\end{array}$ \\
\hline
\end{tabular}

\begin{tabular}{|c|c|c|}
\hline $\begin{array}{l}\text { Índice de capaci- } \\
\text { dad gerencial del } \\
\text { proceso de trans- } \\
\text { ferencia tecnoló- } \\
\text { gica (ICGPTT) }\end{array}$ & $\begin{array}{l}\text { Cazull Imbert, } \\
2008\end{array}$ & $\begin{array}{l}\text { ICGPTT }=\frac{1}{4} \times \sum_{j=1}^{m} P_{j} \times \sum_{i=1}^{n}\left(G_{i} \times P_{i}\right) \\
P_{j}: \text { Peso relativo de cada dimensión de la capacidad gerencial } \\
0 \leq P_{j} \geq 1 . \\
P_{i}: \text { Peso específico de cada factor } 0 \leq P_{i} \geq 1 . \\
G_{i}: \text { Comportamiento de cada factor en la empresa analizada } \\
(0-4) . \\
n: \text { Cantidad de factores de la capacidad gerencial en la transferencia } \\
\text { tecnológica. } \\
m \text { :Cantidad de grupos de dimensiones. }\end{array}$ \\
\hline $\begin{array}{l}\text { Índice de gestión } \\
\text { de la capacidad } \\
\text { tecnológica } \\
\text { (Cgi) }\end{array}$ & $\begin{array}{l}\text { Brito Viñas } \\
2000\end{array}$ & $\begin{array}{l}C g i=\frac{1}{4} \times \sum_{i=1}^{n}\left(K_{g i} \times P_{g i}\right) \\
P_{g i}: \text { Peso relativo de cada indicador de la gestión de la capacidad } \\
\text { tecnológica. } \\
K_{g i}: \text { Comportamiento del indicador en la empresa analizada. } \\
n \text { :Cantidad de indicadores de gestión de la capacidad tecnológica. }\end{array}$ \\
\hline
\end{tabular}




\begin{tabular}{lll}
\hline $\begin{array}{l}\text { Índice compues- } \\
\text { to de competiti- } \\
\text { vidad relativa }\end{array}$ & $\begin{array}{l}\text { Ibarra Mirón, } \\
\left(I C R_{p}\right)\end{array}$ & $I C R_{p}=\sum_{d=1}^{n} I N A_{d} \times W_{d} \times 100 \forall p: p=1 \ldots f e n \%$ \\
& en la dimensión competitiva $(d)$ \\
& $W_{d}:$ Peso de importancia relativa de la dimensión competitiva \\
& $(d)$ para el mercado de actividad \\
& $n:$ Número de dimensiones/variables competitivas $(d)$ implicadas \\
& en la evaluación. \\
& \\
\end{tabular}

Generalmente se utiliza una escala de valoración para determinar en qué estado se encuentra la organización objeto de estudio a continuación se muestran algunas de estas escalas.

Tabla 9. Escala Hedónica (Nogueira Rivera, 2002).

\begin{tabular}{lc}
\hline \multicolumn{1}{c}{ Clasificación } & \multicolumn{1}{c}{ Escala } \\
\hline Muy bueno & $0.80-1.00$ \\
Bueno & $0.60-0.79$ \\
Regular & $0.40-0.59$ \\
Malo & $0.20-0.39$ \\
Muy malo & $0.00-0.19$ \\
\hline
\end{tabular}

Tabla 10. Propuesta de escala (Hernández Nariño, 2010).

\begin{tabular}{lc}
\hline \multicolumn{1}{c}{ Clasificación } & Escala \\
\hline Excelente & $0.80-1.00$ \\
Buena & $0.60-0.80$ \\
Regular & $0.40-0.60$ \\
Mala & $0.20-0.40$ \\
Pésima & $0.00-0.20$ \\
\hline
\end{tabular}


Tabla 11. Escala de valoración de intervalo (Piloto Fleitas, 2011).

\begin{tabular}{cc}
\hline \multicolumn{1}{c}{ Escala } & Clasificación \\
\hline $\mathbf{0 - 0 . 3 3}$ & Desfavorable \\
$\mathbf{0 . 3 3 - 0 . 6 6}$ & Intermedia \\
$\mathbf{0 . 6 6 - 1}$ & Favorable \\
\hline
\end{tabular}

Las ventajas de la determinación de estos índices son indudables, no obstante con su aplicación hasta este momento no garantizan exigencias del Control de Gestión moderno como es la búsqueda de las causas generadoras de las desviaciones (relación causa efecto), ni el carácter proactivo.

La primera de las limitaciones puede ser abordada con la combinación y adecuación de dos herramientas existentes en la literatura: El Método Dupont y el de las Sustituciones Seriadas. El Método Dupont lleva la expresión del indicador (Rentabilidad en su aplicación original) a su mínima expresión, es decir, descompone la expresión; mientras que las Sustituciones Seriadas como su nombre indican sustituye término a término y determina si este cambio provoca una desviación positiva o negativa. La segunda de las limitaciones puede ser resuelta con la utilización de métodos de pronóstico basados en Series Históricas, herramienta utilizable incluso sobre la aplicación del método Dupont mencionado. Medina León et al. (2005) muestra un ejemplo de esta aplicación. 
Figura 1. Método Dupont (Desglose del indicador en forma de árbol para llegar al origen)

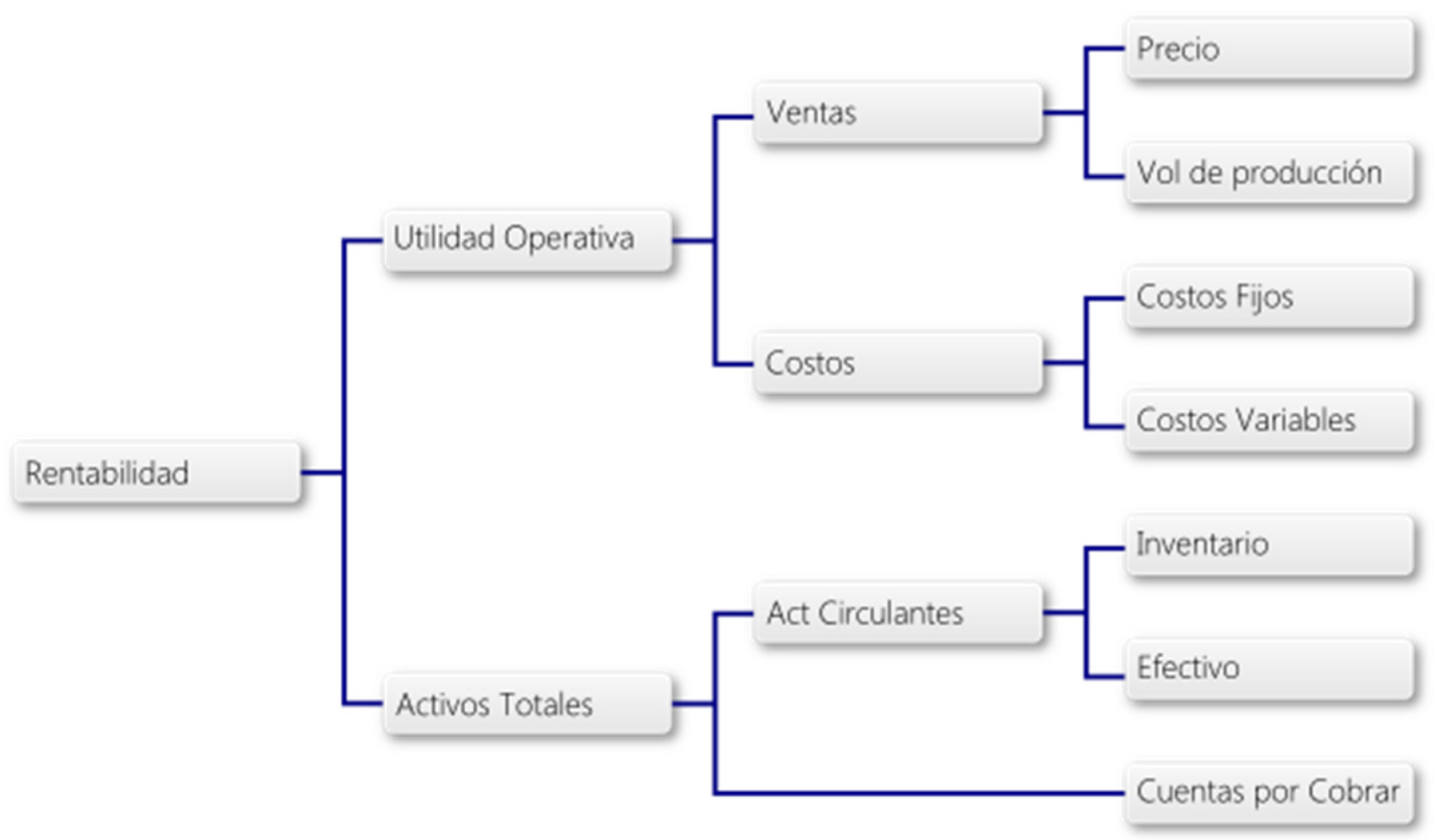

Tabla 12. Método de Sustituciones Seriadas (Analiza el indicador con todos sus factores en la realidad y el plano)

\begin{tabular}{|c|c|c|c|c|c|c|c|c|c|}
\hline & $C F$ & $C V$ & $V p$ & $\boldsymbol{P}$ & $A f$ & $A c$ & Rentabilidad & Diferencia & Influencia \\
\hline Plan & $p$ & $p$ & $p$ & $p$ & $p$ & $p$ & \# & \# & $+o-$ \\
\hline$C F$ & $r$ & $p$ & $p$ & $p$ & $p$ & $p$ & \# & \# & $+o-$ \\
\hline$C V$ & $r$ & $r$ & $p$ & $p$ & $p$ & $p$ & \# & \# & $+o-$ \\
\hline$V p$ & $r$ & $r$ & $r$ & $p$ & $p$ & $p$ & \# & \# & $+o-$ \\
\hline$P$ & $r$ & $r$ & $r$ & $r$ & $p$ & $p$ & \# & \# & $+o-$ \\
\hline $\boldsymbol{A f}$ & $r$ & $r$ & $r$ & $r$ & $r$ & $p$ & \# & \# & $+o-$ \\
\hline$A c$ & $r$ & $r$ & $r$ & $r$ & $r$ & $r$ & \# & \# & $+o-$ \\
\hline
\end{tabular}

Dónde:

CF: $\quad$ Costo Fijo

CV: $\quad$ Costo Variable 
Vp: Volumen de Producción

$P: \quad$ Precio

Af: $\quad$ Activos Fijos

Ac: Activos Circulantes

$p: \quad$ Plan

$r: \quad$ Real

$+o-$ : Positiva o negativa

Un esquema simplificado de los pasos y herramientas recomendadas se muestra en la figura 2, la que sintetiza el procedimiento propuesto. 


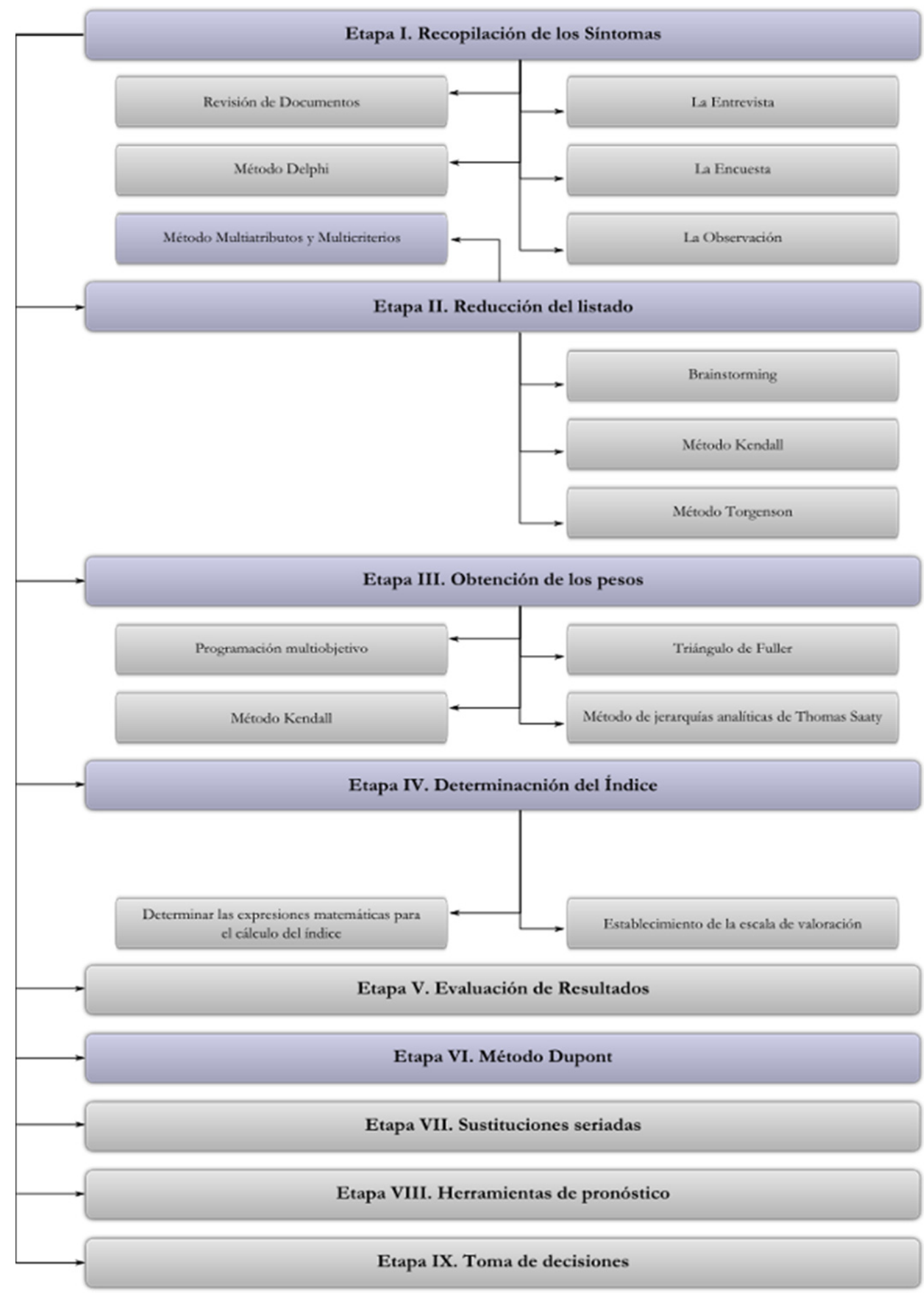

Figura 2. Procedimiento para la construcción de Índices Integrales para el apoyo al Control de Gestión Empresarial. 


\section{CONCLUSIONES}

La creación de Índices Integrales posee un conjunto de pasos comunes los que se pueden resumir en: recopilación de los síntomas, reducción del listado, obtención de los pesos, determinación del índice.

La literatura especializada muestra un arsenal de técnicas para la ejecución de cada uno de los pasos generales, como para la obtención de los síntomas: las entrevistas, la observación, la encuesta, la revisión de Documentos, Método Delphi, Brainstorming.

Para la determinación de los pesos, en el presente trabajo, se mostraron los más comúnmente encontrados en la literatura especializada y que, por demás poseen software que permiten su ejecución. Estos son: Triángulo de Füller y Método de las Jerarquías analíticas (AHP).

La determinación de los Índices Integrales se obtiene por la elaboración de una expresión, en la que se realiza una comparación entre el máximo nivel que puede ser alcanzado, en el caso de que todos los indicadores obtengan la más alta puntuación, y el que posee la organización en la actualidad.

\section{RECOMENDACIONES}

Elaborar un software capaz de integrar los métodos que complementan el procedimiento propuesto como apoyo a la construcción de nuevos Índices Integrales de apoyo al Control de Gestión Empresarial.

\section{BIBLIOGRAFÍA}

Beerli Palacios, A. (2000): "El proceso de la investigación comercial”. Material docente editado para el programa de Maestría en Gestión Turística por la Universidad de Las Palmas Gran Canaria". Plaza América. Varadero (julio). Cuba

Brito Viñas, B. C. (2000). Modelo conceptual y procedimientos de apoyo a la toma de decisiones para potenciar la función de Gestión Tecnológica y de la innovación en la empresa manufacturera cubana. Resumen de la tesis presentada en opción al grado científico de Doctor en Ciencias Técnicas; Universidad Central "Marta Abreu” de las Villas. Santa Clara.

Companys Pascual, R. (1989): Planificación y Programación de la Producción Marcombo, S. A. España.

Cazull Imbert, M. (2008). Gestión de la transferencia de tecnología. Tesis presentada en opción del Grado de Doctor en Ciencias Técnicas. Universidad de Holguín. 
Cuétara Sánchez, L. (2009): Metodología cuantitativa y cualitativa para la evaluación sostenible de destinos y productos turísticos. Revista Retos Turísticos. Cuba, 2009.

Diéguez Matellán, E. (2008): Contribución a la planificación de servicios complementarios extrahoteleros en destinos turísticos. Tesis presentada en opción del Grado de doctor en ciencias Técnicas. Universidad de Matanzas, Cuba.

Ibarra Mirón, S. (2003): Modelo y procedimientos para el análisis y proyección competitiva de unidades estratégicas de fabricación. Tesis presentada en opción del Grado de Doctor en Ciencias Técnicas. Universidad Central "Marta Abreu” de Las Villas.

Hernández Maden, R. et al. (1999) ¿Cómo realizar un diagnóstico logístico de distribución? Revista de la Sociedad Cubana de Logística. No 6 Edit. CNEE. Cuba.

Hernández Nariño, A. (2010): Contribución a la gestión y mejora de procesos en instalaciones hospitalarias del territorio matancero. Tesis presentada en opción del Grado de doctor en ciencias Técnicas. Universidad de Matanzas, Cuba.

Marrero Delgado, F. (2001): Procedimiento para la toma de decisiones logística con enfoque multicriterio. Tesis presentada en opción del Grado de doctor en ciencias Técnicas. Universidad Central "Marta Abreu" de Las Villas.

Marrero Fornaris, C. E. (2002). Diseño de la tecnología integral para la gestión de la formación en instalaciones hoteleras. Tesis presentada en opción del Grado de Doctor en Ciencias Técnicas. Universidad Central "Marta Abreu" de Las Villas.

Medina León, A. et al. (2005): El Control de gestión y su dimensión económica para el sector hotelero. Revista Retos Turísticos. ISSN: 1681- 9713. Universidad de Matanzas, Cuba.

Medina León, A et al (2002). Técnicas de Análisis Empresariales en la Certeza e Incertidumbre. Editora FeGoSa. Michoacán Méjico.

Medina León, A. et al. (2005): El Control de gestión y su dimensión económica para el sector hotelero. Revista Retos Turísticos. ISSN: 1681- 9713. Universidad de Matanzas, Cuba.

Negrín Sosa, E (2003). El mejoramiento de la Administración de Operaciones en Empresas de Servicios Hoteleros. Tesis presentada en opción al grado científico de Doctor en Ciencias Técnicas. Universidad de Matanzas "Camilo Cienfuegos". Facultad Industrial-Economía. Matanzas Cuba. 
Nogueira Rivera, D. (2002). Modelo Conceptual y herramientas de apoyo para potenciar el Control de Gestión en las empresas cubanas. Tesis presentada en opción al grado científico de Doctor en Ciencias Técnicas. Instituto Superior Politécnico "José A. Echevarría”. Ciudad de La Habana.

Nogueira Rivera, D et al. (2002). Los procesos internos y la dimensión financiera del control de gestión. Revista Ciencias Empresariales, No. 12, (julio-diciembre). Facultad de Contabilidad y Administración. Universidad Michoacana de San Nicolás de Hidalgo. Morelia, México.

Nogueira Rivera, D, et al. (2004). Fundamentos para el Control de Gestión Empresarial. Editorial pueblo y Educación. La Habana, Cuba.

Nogueira Rivera, D, \& Medina León, A. (2003): Herramientas de apoyo para el establecimiento del Control de Gestión Moderno en instalaciones Hoteleras. II Simposio Internacional "Turismo y Desarrollo“ TURDES 2003. Universidad de Matanzas, 21 al 25 de Octubre del 2003. ISBN: 959-16-0229-4.

Pérez Campana, M. (2005): Contribución al control de gestión en elementos de la cadena de suministros. Tesis presentada en opción del Grado de doctor en ciencias Técnicas. Universidad Central "Marta Abreu" de Las Villas.

Piloto Fleitas, N. (2011): "Índice de Evaluación Ergonómico de las camareras de piso del sector hotelero". Tesis presentada en opción al título de master en ciencias. Universidad de Matanzas "Camilo Cienfuegos". Facultad Industrial-Economía. Matanzas Cuba.

Real Pérez, G. R: (2011): Modelo y procedimientos para la intervención ergonómica en las camareras de piso del sector hotelero. Caso Varadero, Cuba. Tesis presentada como pre defensa en opción del Grado de Doctor en Ciencias Técnicas. Universidad de Matanzas, Cuba.

Romero, C. (1996): Análisis de las decisiones multicriterio. Editorial ISDEFE, España.

Suárez Mella, R et al. (2001). El Reto. Gestión de vitalidad en entornos competitivos; Editorial Academia, Ciudad de la Habana. Cuba.

Suárez Mella, R. (1995): Modelo de evaluación del nivel organizativo para empresas industriales. Ponencia presentada en el seminario internacional de ciencias empresariales. Universidad de Matanzas. 
Sarache Castro, W. A. (2003): Modelo con enfoque estratégico y procedimiento para contribuir al incremento del nivel de desempeño de las PyME’s de confección desde la función de producción. Tesis presentada en opción del Grado de Doctor en Ciencias Técnicas. Universidad Central "Marta Abreu" de Las Villas.

Suarez Hernández, J. (2003): Modelo general y procedimientos de apoyo a la toma decisiones para desarrollar la Gestión de la Tecnología y de la innovación en empresas ganaderas cubanas. Tesis presentada en opción del Grado de doctor en ciencias Técnicas. Universidad de Matanzas, Cuba. 